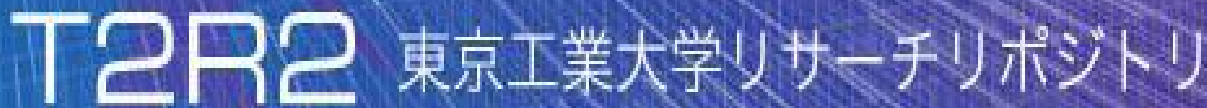

\section{Tokyo Tech Research Repository}

\section{論文 /著書情報 \\ Article /Book Information}

\begin{tabular}{|c|c|}
\hline Title & $\begin{array}{l}\text { Using hyperspectral image enhancement method for small size object } \\
\text { detection on the sea surface }\end{array}$ \\
\hline Authors & $\begin{array}{l}\text { Lu Yan, Masahiro Yamaguchi, Naoki Noro, Yohei Takara, Fuminori } \\
\text { Ando }\end{array}$ \\
\hline Citation(English) & Proc. SPIE, Vol. 9643, , 96430H, 9 pages \\
\hline 発行日 /Pub. date & 2015,10 \\
\hline DOI & http://dx.doi.org/10.1117/12.2194606 \\
\hline 権利情報 /Copyright & $\begin{array}{l}\text { 本著作物の著作権はSociety of Photo-O ptical Instrumentation } \\
\text { Engineersに帰属します。 } \\
\text { Copyright } 2015 \text { Society of P hoto-O ptical Instrumentation Engineers. } \\
\text { One print or electronic copy may be made for personal use only. } \\
\text { Systematic reproduction and distribution, duplication of any material in } \\
\text { this paper for a fee or for commercial purposes, or modification of the } \\
\text { content of the paper are prohibited. }\end{array}$ \\
\hline
\end{tabular}




\title{
Using hyperspectral image enhancement method for small size object detection on the sea surface
}

\author{
Lu Yan*a ${ }^{\mathrm{a}}$, Naoki Noro ${ }^{\mathrm{b}}$, Yohei Takara ${ }^{\mathrm{b}}$, Fuminori Ando $^{\mathrm{b}}$, Masahiro Yamaguchi ${ }^{\mathrm{a}}$ \\ ${ }^{a}$ Interdisciplinary Graduate School of Science and Engineering, Tokyo Institute of Technology, \\ 4259-G2-28 Nagatsuta-cho, Midori-ku, Yokohama, Japan 226-8503; \\ ${ }^{b}$ EBA JAPAN CO.,LTD., 2-17-12-301, Takanawa Minato-ku, Tokyo, Japan 108-0074
}

\begin{abstract}
Small size object detection in vast ocean plays an important role in rescues after accident or disaster. One of the promising approach is a hyperspectral imaging system (HIS). However, due to the limitation of HIS sensor's resolution, interested target might occupy only several pixels or less in the image, it's difficult to detect small object, moreover the sun glint of the sea surface make it even more difficult. In this paper, we propose an image analysis technique suitable for the computer aided detection of small objects on the sea surface, especially humans. We firstly separate objects from background by adapting a previously proposed image enhancement method and then apply a linear unmixing method to define the endmember's spectrum. At last, we use spectral angle mapping method to classify presented objects and thus detect small size object. The proposed system provides the following results for supporting the detection of humans and other small objects on the sea surface; an image with spectral color enhancement, alerts of various objects, and the human detection results. This multilayered approach is expected to reduce the oversight, i.e., false negative error. Results of the proposed technique have been compared with existent methods, and our method has successfully enhance the hyperspectral image, and detect small object from the sea surface with high human detection rate, shows the ability to further detection of human in this study). The result are less influenced by the sun glint effects. This study helps recognizing small objects on the sea surface, and it leads to advances in the rescuing system using aircraft equipped HIS technology.
\end{abstract}

Keywords: hyperspectral image, principal component analysis, spectral angle mapping, vertex component analysis, object detection

\section{INTRODUCTION}

Compared to traditional color images used in various imaging application systems, hyperspectral images (HSIs) have the advantage of providing both spatial and spectral information in high-resolution. Based on such advantages, hyperspectral images have been increasingly used in various fields such as agriculture, oceanology, geology, etc. Among different applications, object detection and classification have always been an important task of HSI. Normally object detection algorithms propose to construct a detector based on assumption that data set follow a pre-known probability distribution. Nowadays, some object reflectance spectrum dataset have been published and could be use freely for scientific researches, such as USGS Digital Spectral Library, ASTER Spectral Library, etc. However those databases only cover a minority part of existent object. According to real applications, the pre-knowledge of site presented object are difficult to obtain. Moreover, in most case of HSI, the object are very tiny compared to the background size, thus it make the problem more difficult to solve.

Human search and rescue mission have always been an important and difficult issue because of its immediacy and accuracy. Sumimoto et al. ${ }^{1}$ proposed a methods using color information and the composite image sensor to detect the human object in the sea area. Their methods need pre-knowledge of human object like color, size or shape, however for most of the case, such information cannot be provided properly due to various cases. Westall et al. ${ }^{2}$ have studied several different point target detections techniques and tracking techniques for aerial search of humans applying different color spaces images. Their results show the possibility to use machine in the maritime human search application. On the other hand, the system is not yet suitable for real search operations due to the false alarm for an acceptable missed detection rate. Doherty and Rudol ${ }^{3}$ propose an Unmanned Aerial Vehicles search and rescue scenario for human body detection. In their proposed method, they using thermal image to find potential human body temperature alike region. Then certain

Image and Signal Processing for Remote Sensing XXI, edited by Lorenzo Bruzzone, Proc. of SPIE Vol. 9643, 96430H · C 2015 SPIE · CCC code: 0277-786X/15/\$18 · doi: 10.1117/12.2194606 
region which not resemble to human shape will be rejected. Corresponding pixel in the color image will be identified and using a classifier which been trained with Haar-like features. Their results shows feasibility of such system, however this kind of system need large dataset of training data and no evidence shows can be used for other rescue situation such as marine. The existence methods need pre-knowledge of human shape or other information, the detection results could be various if the training dataset is not well selected or not enough.

The application of HSI is promising as mentioned above, but there are several issues that need to be solved for realizing a practical system. The target object, such as human or boat, is very small, interested target might occupy only several pixels or less in the image, it is difficult to detect smalls object by applying a pattern matching technique. In addition, the sun glint on the sea surface disturbs the discrimination of human and other objects. It is expected to enable the classification of pixels based on spectral information, but the direct detection of the spectral signature of human is difficult, because human is wearing clothes and the spectral signature is mixed. Moreover, the sunlight spectrum is not calibrated, and the background reflection varies depending on the sunlight, sea depth, etc.

In our study, for the purpose to eliminate the variation of sunlight and background spectra, we apply partly a multispectral color enhancement method proposed by Mitsui et al. ${ }^{4}$, in which they enhance the remaining part apart from the several significant principal components and then merge the enhance image to original image. Hashimoto et al. ${ }^{5}$ improve this method for more effective visualization of the spectral features to non-visible range. Their methods have successfully visualize the spectral features that need to be enhanced even when the background scene has spectral variations. In our study, we apply the method to restrain the background information. Then, for the purpose to detect small objects, we utilize a linear unmixing technique ${ }^{6}$ to find the endmembers, and the derived endmember spectra are classified into human and other objects using spectral angular mapping (SAM) ${ }^{7}$.

The rest of the paper is organized as follows: in Section 2 the mainly technics applied in our study is introduced; then Section 3 the martials and proceeding is described; in Section 4 the results obtained by the proposed scheme on a real HSI data set are presented and discussed before draw conclusion in Section 5.

\section{DETECTION METHOD}

\subsection{Overview of the proposed method}

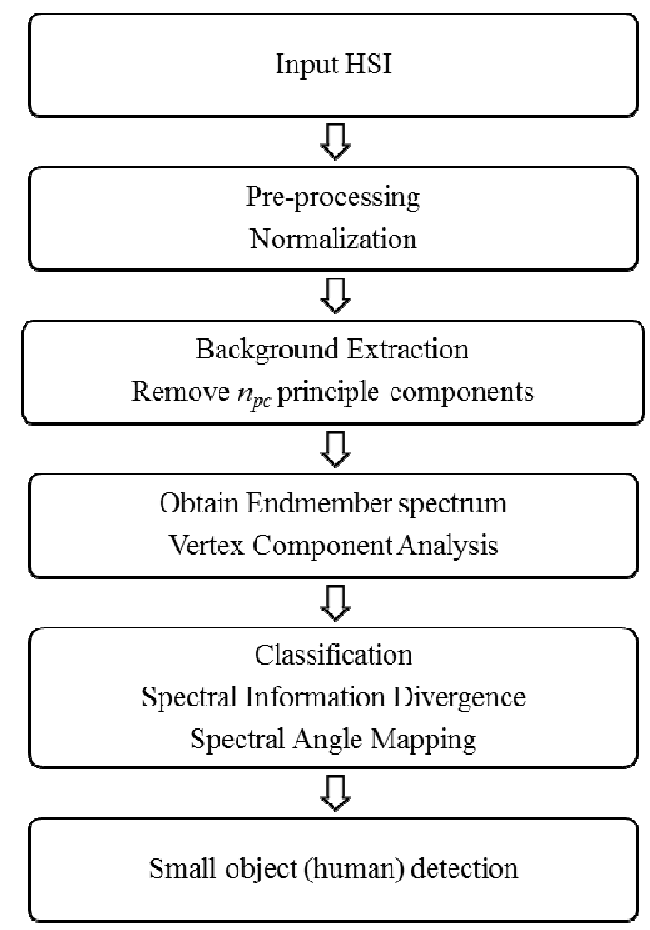

Figure 1. Proposed processing chain 
The overview of the proposed detection method is shown in Figure 1. First of all, we normalize the data, then background information is been removed. Based on the background removed data, each endmember's spectra is been extracted and then different classification methods have been applied. At last, depending on the discrimination map of each endmember, and the human spectra characteristics, a specific classification rule is defined for the human detection.

\subsection{Background separation}

Principal component analysis (PCA) have been often used for dimension reduction: the projection data according to its contribution, The first principal component have contain the direction that data mostly distributed, the second principal component orthogonal to the first ones and follow the direction that data mostly distributed apart from the first principal component. Thus, the first several principal components contain most of information of the dataset. In the case of HSI of sea surface, they contain most of the background information.

In our study, we would like to focus on the human and other objects that suggest the presence of human (which take only small part of image) by reducing the background information. By deleting the first several principal components from the original data, we can obtain the HSI which has its background reduced. The HSI data cube containing H rows, W columns and $\mathrm{N}$ bands (a three-dimensional data cube $\mathrm{H}^{*} \mathrm{~W} * \mathrm{~N}$ ), $\mathrm{s}_{0}(\mathrm{~h}, \mathrm{w})$ is a column vector of the spectrum from spatial coordinates $(\mathrm{h}, \mathrm{w}), \mathrm{w}=1,2, \ldots, \mathrm{W}, \mathrm{h}=1,2, \ldots, \mathrm{H}$. After PCA, note $\boldsymbol{u}_{\mathrm{i}}$ be the $i$-th eigen vector, thus by forcing the first $n$ principal components to be zero, background-suppressed spectrum $\boldsymbol{s}_{\mathrm{br}}$ can be obtained.

$$
\mathbf{s}_{b r}(h, w)=\left(\mathbf{0}, \mathbf{0}, \ldots \mathbf{u}_{n+1} \ldots \mathbf{u}_{N}\right)\left(\begin{array}{l}
\mathbf{0} \\
\mathbf{0} \\
\vdots \\
\mathbf{u}_{n+1}{ }^{t} \\
\vdots \\
\mathbf{u}_{N}{ }^{t}
\end{array}\right) \mathbf{s}_{0}(h, w) .
$$

\subsection{Endmember spectra extraction}

Linear spectral unmixing methods consider that a mixed pixel is the linear combination of endmember signatures. Many algorithm have been proposed for spectral unmixing ${ }^{10}$. Most of the linear spectral unmixing model assume that endmembers are presented in the scene. That is to say in the HSI, at least exist one pure pixels for each endmember. Vertex component analysis $(\mathrm{VCA})^{6}$ is based on the convex sets. It finds the vertices of the simplex after affine transformation. The obtained vertices are considered to be the endmembers. Compared to other linear spectral unmixing methods, VCA have simpler or even better performance while at the same time its computational complexity are the lowest. Thus in our study we apply this methods to determine the endmember's spectra.

\subsection{Classification}

After the spectra of endmembers are obtained, we need to classify the HSI in order to detect the objects. There are many different way in hyperspectral image classifications: Maximum likelihood(MLC), artificial neural network(ANN), Support vector machine(SVM) and Spectral match classifier such as Spectral angle mapping(SAM) and spectral information divergence(SID).

MLC have been generally applied in multispectral image classification, however, for HSI applications the calculation time is significantly increased. ANN is similar when applied to HSI, the iteration time increased. SVM gives promising result, however it need to carefully choose the kernel function and other parameters. SAM and SID are easy and fast to calculate, thus they are very suitable for real-time proceeding system.

Suppose two pixels that have spectral data: 
$\mathrm{X}=\left(x_{1}, x_{2}, \cdots x_{n}\right)^{T}, \mathrm{Y}=\left(y_{1}, y_{2}, \cdots y_{n}\right)^{T}$

Spectral angle $\alpha$ is expressed as

$$
\cos (\alpha)=\frac{\mathrm{X} \bullet \mathrm{Y}}{\|\mathrm{X}\|\|\mathrm{Y}\|}
$$

where $\alpha \in\left[0, \frac{\pi}{2}\right]$

When $\alpha$ is small, that shows $\mathrm{X}$ and $\mathrm{Y}$ similar.

Spectral Information divergence, $\operatorname{SID}(X, Y)$ is the divergence of two spectral to estimate their similarities, given by

$$
\operatorname{SID}(X, Y)=D(x \| y)+D(y \| x)
$$

where

$$
D(x \| y)=\sum_{i=1}^{N} p_{i} \log \frac{p_{i}}{q_{i}} \quad D(y \| x)=\sum_{i=1}^{N} q_{i} \log \frac{q_{i}}{p_{i}}
$$

and

$$
p_{i}=\frac{x_{i}}{\sum_{i=1}^{N} x_{i}}
$$

$$
q_{i}=\frac{y_{i}}{\sum_{i=1}^{N} y_{i}} .
$$

In the proposed method, Spectra angle and SID have been calculated for each pixel compared to the endmembers obtained in section 2.3

\section{EXPERIMENT}

\subsection{Materials}

We use a set of HSI provided by EBA-JAPAN CO., LTD. Images are taken using their NH series hyperspectral camera ${ }^{11}$, which is a pushbroom-type sensor. Each HSI is 1024 pixels (height) by 2048 pixels (width), from 400nm to $1000 \mathrm{~nm}$ with interval of $5 \mathrm{~nm}$, totally 121 channels. Each HSI is a scene of surfboard floating on the sea with human on or around as shown in Figure 2. Here, we select a sub-region size 600 by 600 for better presented the result. The sub-region we selected include 11 surfboard. Some human have presented on the surfboard (the left upper corner) while some of them are not (the right downer corner), the absent human believe to exist around the board, might be in the water. Therefore total 11 human object need to detect in this sub-region we selected.

\subsection{Processing}

We firstly extract the interested area from the original HSI data cube, then apply normalization to bring the digital value to the range $[0,1]$ by subtracting the minimum digital value and then divided by the range of the digital value. 


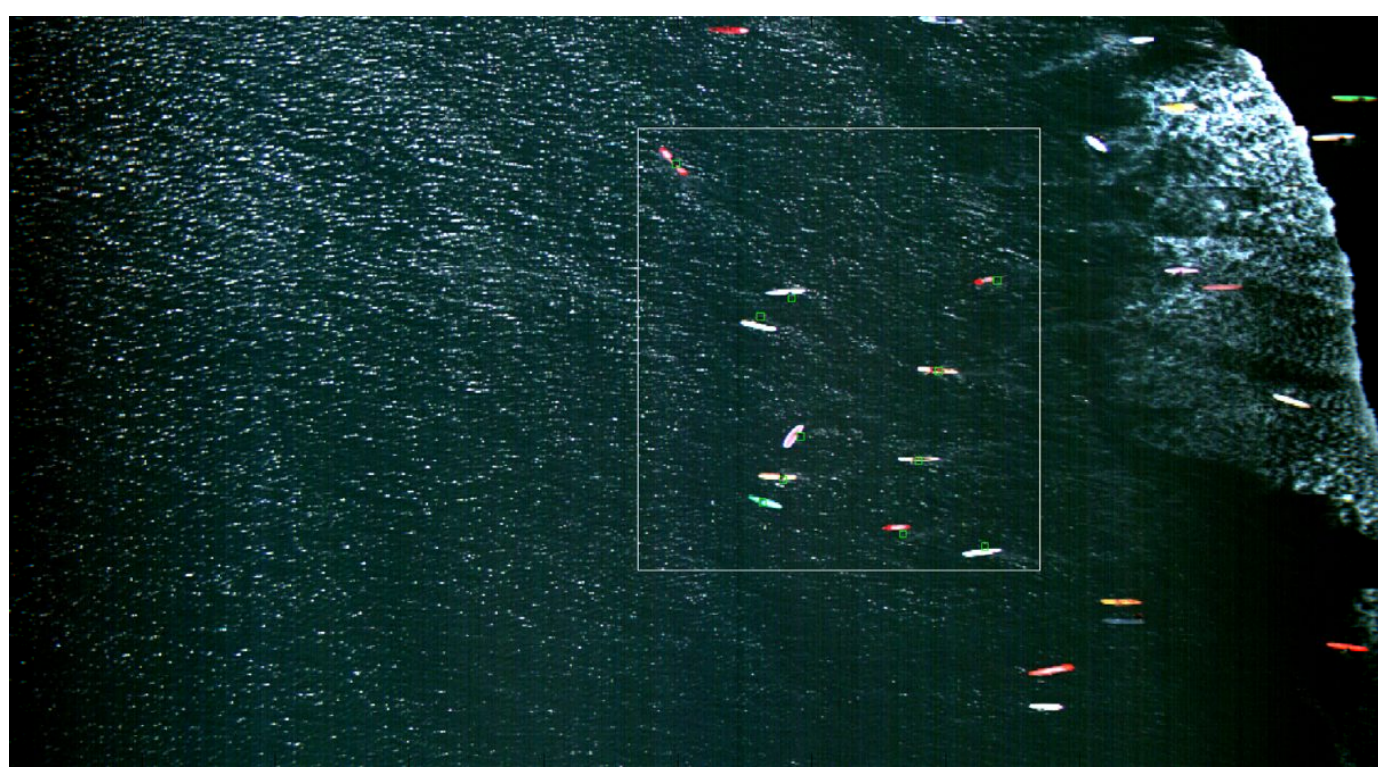

Figure 2. HSI used in this study, sub-region within the white rectangle are the testing area. Human positions have been marked as green rectangles.

Secondly, using equation 1 , we can remove the first $n$ principal components. In order to define the value of $n$, the cumulative contribution of principal component is plotted in Figure 3. As we can see, the first 3 principal components have cumulative contribution about $90 \%$, we define n's value for this dataset is 3 .

Next we need to define the number of endmembers, since there is totally 11 surfboard in the scene, and consider human spectrum, sea spectrum, as well as sea glint spectrum, we define 14 endmembers to be unmixed by applying VCA.

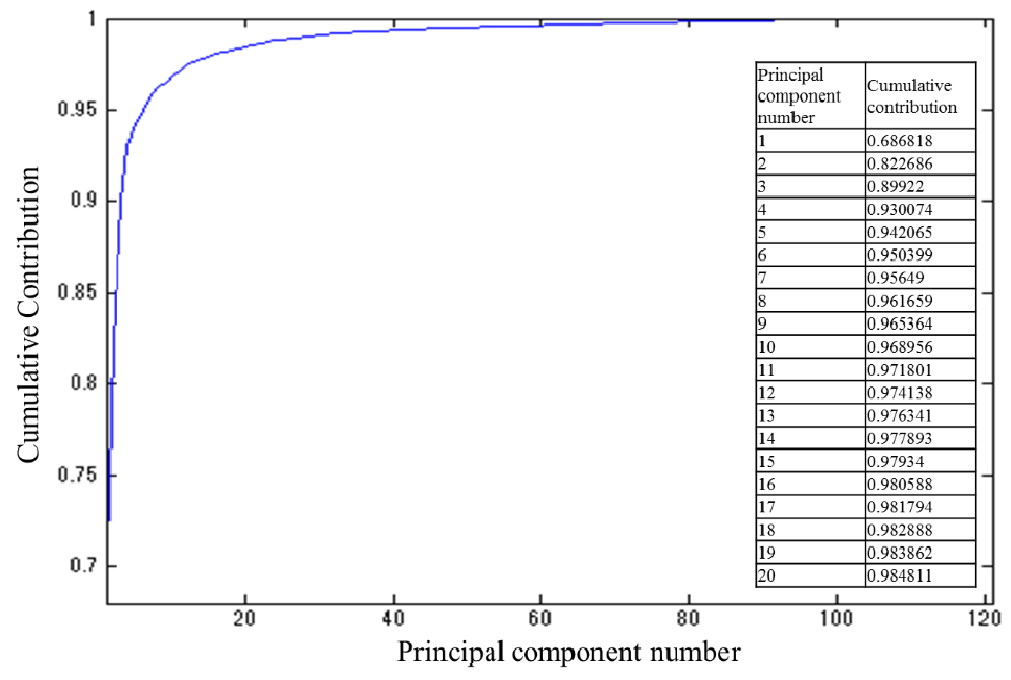

Figure 3. Principal component cumulative contribution

Once we obtain the endmembers' spectrum, we calculate spectral angle and SID for each pixel compared with each endmember's spectrum to get the abundance map. After that, we select 2 or 3 abundance images and set certain threshold to obtain the final results which present in next section. 


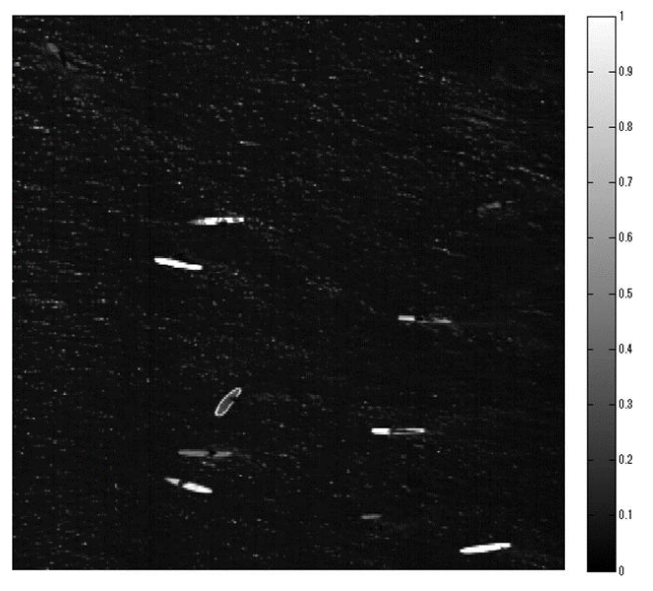

(a)

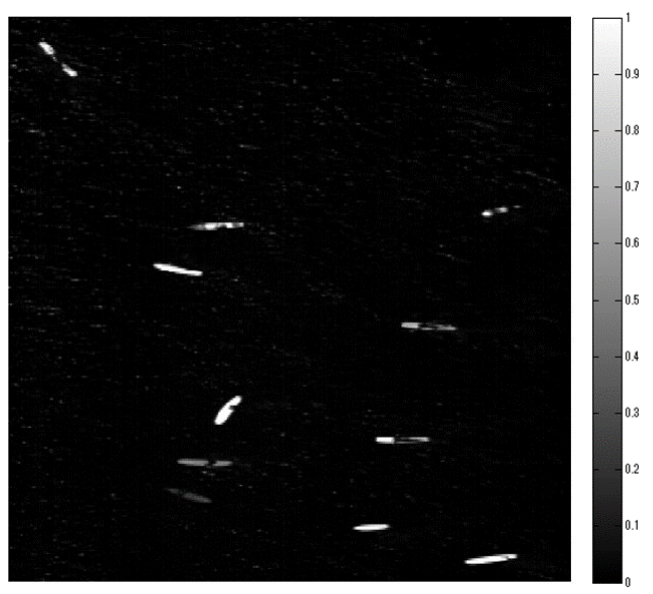

(b)

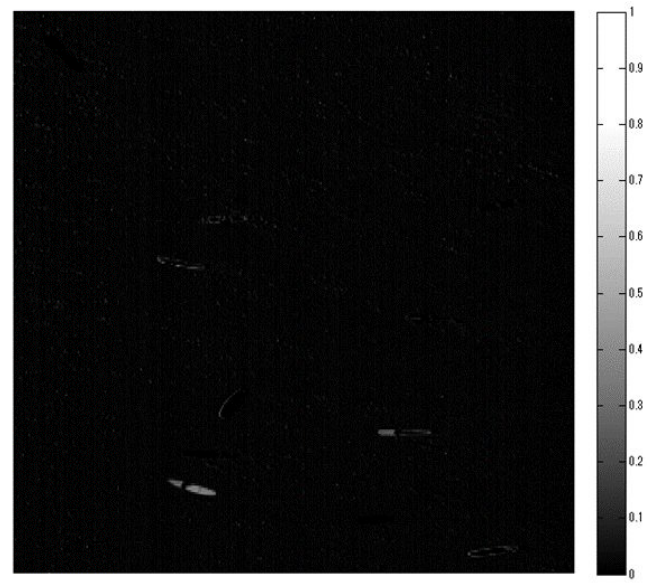

(c)

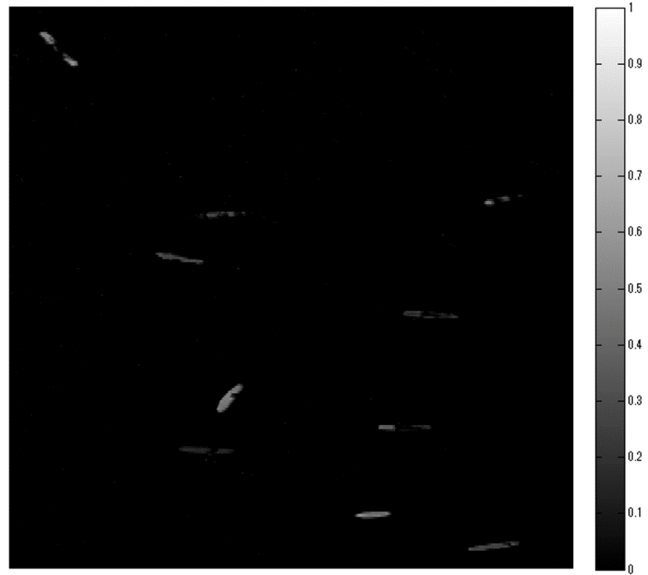

(d)

Figure 4. HSI images at wavelength .(a).495 nm and (b)695 nm; images after remove first 3 principal components at wavelength (c) $495 \mathrm{~nm}$ and (d) $695 \mathrm{~nm}$

\section{RESULTS}

In order to constrain the background information, we have deleted the first 3 principal components then reconstruct our spectrum. Shown in Figure 4, (a) is the original HSI sub-image at $495 \mathrm{~nm}$, we can see clearly the vast distribution of sun glint. Compared with (c) which is after background information constrain, we can almost see no sun glint. For $695 \mathrm{~nm}$ is the similar situation.

Figure 5 (a) shows the normalized spectral of objects presented in the images, while Figure 5 (b) shows the endmember spectra results obtained by the VCA. As we can observe, the $7^{\text {th }}$ endmember extracted characteristically like the Human spectra, and many endmember almost the same as surfboard spectra respectively. Results shows the ability of VCA methods to determinate endmember although some objects spectra might not be well extracted, this might due to the saturation of data in spectra.

We compared our methods with and without background constrain, and also compare our result with RX (Reed-Xiaoli) detecting method ${ }^{5,6}$. In the proposed method, SID and SAM are used in the classification step. Results have been shown in Figure 6.

The detection results shown in Table 1. Overall, the RX detector method can detect all the surfboard, however poor performance on human detection. It might cause by the human spectrum more similar to the sea surface other than surfboard. The spectrum methods although don't detect all the boat, they perform better on human detection. This might be caused by the spectrum unmixing method that have obtain the human spectrum signature. SID performance is better 
in human detection, however at the same time its false alarm numbers are extremely high. Therefore it always trade-off of better detection rate and higher false alarm rate. By applying background suppression, false alarm rate have been significantly decreased: for RX method and SAM method, the false alarm both eliminated, even for SID method, it have been reduced to about $42 \%$.

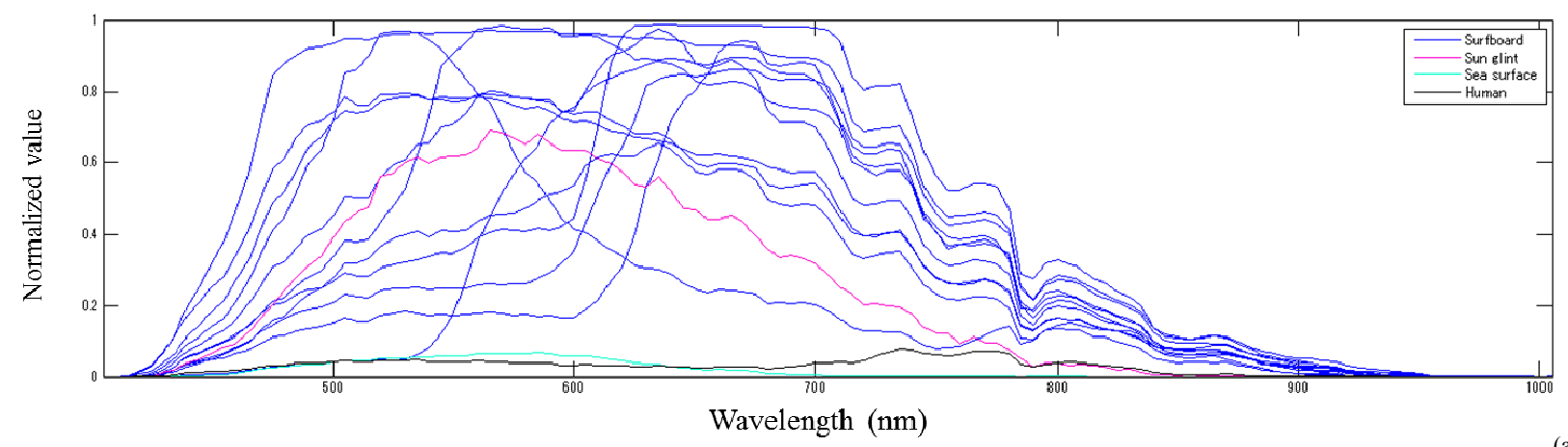

(a)

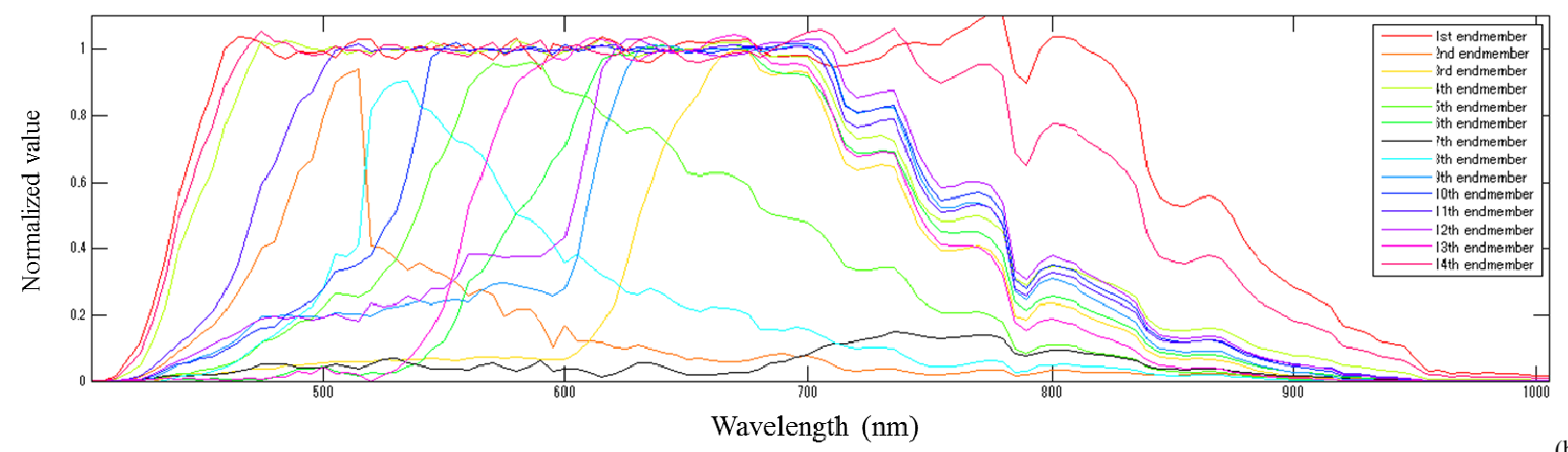

Figure 5. (a) Original Spectral of Objects presented in the scene. (b) Endmember obtained by VCA.

Table 1.Detection results (a) Without background constrain, (b) With background constrain,

\begin{tabular}{|l|c|c|c|c|c|c|}
\hline \multirow{2}{*}{} & \multicolumn{2}{|c|}{ Surfboard Detection } & \multicolumn{2}{c|}{ Human Detection } & \multicolumn{2}{c|}{ False Alarm } \\
\cline { 2 - 7 } & (a) & (b) & (a) & (b) & (a) & (b) \\
\hline RX detector & 11 & 11 & 4 & 3 & 12 & 0 \\
\hline SAM & 9 & 11 & 7 & 4 & 12 & 0 \\
\hline SID & 10 & 9 & 9 & 9 & 38 & 16 \\
\hline
\end{tabular}

\section{CONCLUSION}

In this paper, we proposed a small target detection proceeding method for human detection from aerial HIS of sea surface. That is to firstly apply background constrain and then using spectrum unmixing for classification. Experimental results suggest that SAM method's is similar as RX detector, with improved human detection rate. And both methods have zero false alarm after background suppression. SID method have highest human detection while at the same time have highest false alarm rate. However background suppression enables to greatly reduce SID's false alarm rate, which shows the potential of using SID method for practical human detection. 

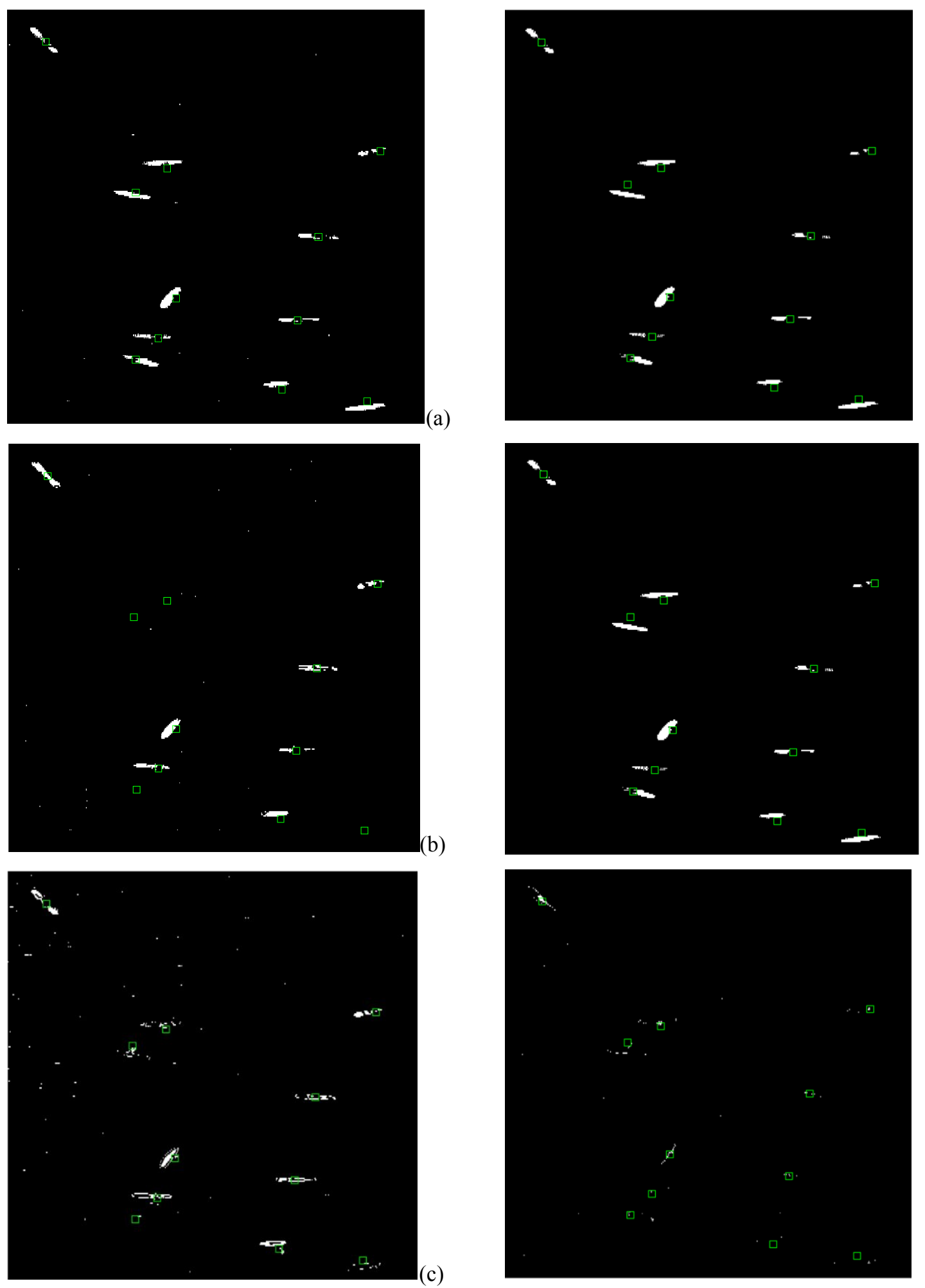

Figure 6. Results for (a) RX detector, (b) SAM classification, (c) SID classification, left: without removing principal components, right: remove first 3 principal components. Green rectangle are the position of human

Proc. of SPIE Vol. $964396430 \mathrm{H}-8$ 


\section{REFERENCES}

[1] Sumimoto, T., Kuramoto, K., Okada, S., Miyauchi, H., Imade, M., Yamamoto, H., T. Kunishi, H., "Machine vision for detection of the rescue target in the marine casualty," Proc. 20th International Conference on Industrial Electronics, Control and Instrumentation 2, 723-726 (1994).

[2] Westall, P., Ford, J. J., O'Shea, P., Hrabar, S., "Evaluation of Maritime Vision Techniques for Aerial Search of Humans in Maritime Environments," Digital Image Computing: Techniques and Applications (DICTA), 176183(2008).

[3] Doherty, P. and Rudol, P., "A UAV Search and Rescue Scenario with Human Body Detection and Geolocalization," AI 2007: Advances in Artificial Intelligence, Proc. 20th Australian Joint Conference on Artificial Intelligence, 113(2007).

[4] Mitsui, M., Murakami, Y., Obi, T., Yamaguchi, M., \& Ohyama, N., "Color enhancement in multispectral image using the Karhunen-Loeve transform," Optical review 12(2), 69-75 (2005).

[5] Hashimoto, N., Murakami, Y., Bautista, P. A., Yamaguchi, M., Obi, T., Ohyama, N., and Kosugi, Y., "Multispectral image enhancement for effective visualization," Optics express 19(10), 9315-9329 (2011).

[6] Nascimento, J., Bioucas Dias, J., "Vertex component analysis: a fast algorithm to unmix hyperspectral data," IEEE Trans. Geoscience and Remote Sensing 43(4), 898-910 (2005).

[7] Chang C. I., "Spectral information divergence for hyperspectral image analysis," IEEE Geoscience and Remote Sensing Symposium 1, 509-511 (1999).

[8] I. S. Reed and X. Yu, "Adaptive multiple-band CFAR detection of an optical pattern with unknown spectral distribution," IEEE Trans. Acoustics, Speech, Signal Processing 38, 1760-1770 (1990).

[9] Chang, C. I., and Chiang, S.S., "Anomaly detection and classification for hyperspectral imagery," IEEE Trans. Geoscience and Remote Sensing 40(6), 1314-1325 (2002).

[10] Keshava, N., and J. F. Mustard. "Spectral unmixing," IEEE Signal Processing Magazine 19(1), $44-57$ (2002). 